\title{
Robotic surgery in early-stage endometrial cancer
}

\author{
Giacomo Corrado ${ }^{1}$, Simone Bruni ${ }^{2}$, Enrico Vizza ${ }^{2}$ \\ ${ }^{1}$ Department of Woman, Child Health and Public Health, Gynecologic Oncology Unit, Fondazione Policlinico Universitario A. Gemelli, IRCCS, \\ Rome, Italy; ${ }^{2}$ Department of Experimental Clinical Oncology, Gynecologic Oncology Unit, "Regina Elena” National Cancer Institute, IRCCS, \\ Rome, Italy \\ Correspondence to: Dr. Giacomo Corrado, MD, PhD. Department of Woman and Child Health, and Public Health, Gynaecologic Oncology Unit, \\ Fondazione Policlinico Universitario A. Gemelli, IRCCS, Università Cattolica del Sacro Cuore, Roma, Italy. Email: giacomo.corrado@alice.it. \\ Comment on: Jørgensen SL, Mogensen O, Wu CS, et al. Survival after a nationwide introduction of robotic surgery in women with early-stage \\ endometrial cancer: a population-based prospective cohort study. Eur J Cancer 2019;109:1-11.
}

Submitted Jul 08, 2019. Accepted for publication Aug 05, 2019.

doi: $10.21037 /$ tcr.2019.08.27

View this article at: http://dx.doi.org/10.21037/tcr.2019.08.27

In the past years, the standard of treatment for endometrial cancer was laparotomy with extra-fascial hysterectomy, bilateral salpingo-oophorectomy (BSO), with or without pelvic (PL) and paraaortic lymphadenectomy (PAL); however, many studies have shown that this type of surgery was mainly associated with bleeding, pain, infections and dehiscences especially in diabetic and obese patients (1). Conventional laparoscopy represents one of the main advancements of the last century, since performing very small incisions, surgical interventions similar to those of open surgery can be performed.

A large prospective randomized trial called LAP2, with the aim of comparing the laparotomic with laparoscopic approach regarding the staging of endometrial cancer, showed that laparoscopy was associated with fewer postoperative complications and shorter hospital stay. Furthermore, the estimated 5 -year overall survival was similar in both groups at $89.8 \%$ (2). Therefore, to date traditional laparoscopy is the standard approach for the surgical treatment of endometrial cancer (3). Robotic surgery was born as an advancement of laparoscopic surgery, in fact, the 20th century was characterized by the introduction and diffusion of increasingly advanced minimally invasive surgery approaches.

The use of robots as an aid to surgical practice began in 1980 , but only in the early 2000 the first version of the da Vinci robot was marketed. Since the Food and Drug Administration allowed the da Vinci system for the use in gynaecology in April 2005, the advantages of robotic surgery for gynaecological oncological procedures have quickly emerged.

The ergonomics of the console increases the comfort of surgeon during surgery, while the Endowrist instruments with seven degrees of freedom and $90^{\circ}$ articulation offer significant technical advantages eliminating the fulcrum effect typical of laparoscopy, allowing the surgeon to perform simplified spatial movements, filter the tremor and increase precision of demolitive and reconstructive procedures. The system also provides the surgeon with a stereoscopic view obtained with a two-channel optical video endoscope and dual high-resolution 3CCD camera. This real-time magnification system gives to the surgeon a remarkable quality of image compared to traditional surgery. The three-dimensional vision not only allows to have easier access to difficult anatomies, but it seems to replace in expert hands the tactile sensation of the tissues and the tension exerted that we have in laparoscopy and in open surgery, thus favouring an easy preparation of the anatomical spaces and structures.

In recent years, many branches of surgery in addition to traditional robotic platforms (4) have used new and increasingly efficient robotic systems as single-site robotic platform.

Regarding safety and complications of robotic surgery, a Cochrane review published in 2014 found limited evidence on the safety and effectiveness of robotic surgery compared with laparotomy and traditional laparoscopy for surgical procedures performed for gynaecological cancers (5). Subsequently a review of four previously published meta-analysis involving 2,196 laparoscopic, 
2,913 robotic and 1,219 laparotomy-treated endometrial cancer patients showed similar incidence of complications between laparoscopy and robotic surgery, except for fewer conversions to laparotomy and reduced blood loss for the robotic group (6).

A recent systematic review and meta-analysis have included 24 studies comparing robotic hysterectomy (RH) to open hysterectomy $(\mathrm{OH})$ and 24 studies comparing $\mathrm{RH}$ to laparoscopic hysterectomy $(\mathrm{LH})(7)$. There was less estimated blood loss, the length of hospital stay was shorter and the rates of transfusion, complications and readmission were lower with $\mathrm{RH}$ compared to $\mathrm{OH}$. However, $\mathrm{RH}$ showed a higher incidence of vaginal cuff dehiscence and a longer operative time compared to $\mathrm{OH}$. Compared to $\mathrm{LH}$, there was less estimated blood loss, the length of hospital stay was shorter and the rates of conversion to laparotomy, intra-operative complications, cystotomy and urinary tract injuries were lower in RH. Therefore, robotic surgery could represent a safer therapeutic surgical strategy compared to $\mathrm{LH}$ and $\mathrm{OH}$ for the treatment of endometrial cancer.

Another more recent review and meta-analysis comparing robotic surgery and laparoscopy for endometrial cancer included two matched case-control studies, 33 retrospective studies and one randomized controlled trial, with a total of 8,075 patients $(3,830$ robotic and 4,245 laparoscopic) (8). There were no differences in the duration of surgery but hospital stay, blood loss, conversions to laparotomy and complications were lower in the robotic arm. In a recent RCT, patients with histologically proven endometrial cancer were randomised to hysterectomy, BSO, PL and PAL by either robot-assisted surgery or laparotomic surgery demonstrating non-inferiority in paraaortic lymph node count, comparable complication rates, shorter hospital length and lower total cost for robotic surgery over laparotomy (9). In another recent RCT, 101 endometrial cancer patients were randomized to hysterectomy, BSO and PL either by robotic surgery or by conventional laparoscopy. This study showed that roboticassisted surgery was faster to perform than laparoscopy, that all conversions to laparotomy occurred in the laparoscopic group and that surgical outcome (number of lymph nodes removed, bleeding, length of postoperative hospital stay and complications) was similar between the groups (10).

A group of patients that benefit from robotic surgery are obese patients. Obesity in the past has been considered a contraindication for laparoscopic surgery due to the difficult access to the anatomical spaces and the comorbidities associated with obese patients (cardiovascular and respiratory diseases). Conversely, some studies have showed that laparoscopy provides numerous benefits also in this group of patients, including reduced blood loss, lower wounds infections and reduced risk of embolism (11). Recently, lower conversion rates have been demonstrated for robotic surgery and numerous advantages in terms of reduced surgical times, shorter hospital stay and reduced blood loss compared to traditional laparoscopy in obese women $(12,13)$.

Regarding the oncological outcome, a retrospective study published in 2014 analysed a total of 183 women subjected to robotic surgery and 232 women subjected to laparoscopic surgery from 2003 through 2010 showing no significant differences in overall survival, disease free survival (3-year DFS $83.3 \%$ and $88.4 \%$ ), and tumour recurrence (14.8\% and $12.1 \%)$ for robotic and laparoscopic groups, respectively (14). Moreover, a retrospective chart review was performed for all endometrial cancer patients surgically staged with robotic surgery at the University of North Carolina Hospital from 2005 to 2010, including a total of 499 patients (15). Study results were compared to endometrial cancer statistics from the National Cancer Institute, showing that robotic surgical staging of endometrial cancer is not correlated with inferior results when compared to traditional laparoscopy and laparotomy.

A report from the American College of Surgeons National Surgical Quality Improvement Program database compared surgical outcomes in more than 2,000 surgeries for patients affected by endometrial cancer performed by laparotomy or minimally invasive surgery in the United States between 2006 and 2010 (16). The authors suggested that if $90 \%$ of patients would undergo to minimally invasive surgery, 8,059 fewer complications, 127,257 fewer days of hospitalization and 534 million dollars would be saved yearly (17).

A single-institutional retrospective cohort study performed by National Cancer Institute of Rome compared homogeneous groups of patients who underwent to laparotomic, laparoscopic and robotic surgery for endometrial cancer (18). The study evidenced that minimally invasive surgery was superior to laparotomic surgery in terms of surgical outcomes and that robotic surgery was superior to laparoscopy in terms of intra- and post-operative complications, conversion rates, length of hospital stay and reinterventions while in terms of oncological outcomes (rate of recurrences and survival) the three groups were similar.

Although many of these retrospective studies on robotic 
surgery in endometrial cancer have showed encouraging results regarding survival outcome, new prospective and RCT studies comparing the different surgical approaches are awaited.

For this reason, the prospective study of Jørgensen et al. had the purpose of collecting all the patients who underwent surgery for endometrial adenocarcinoma (FIGO stage I-II) from January 2005 to June 2015 in Denmark, performing the research with the nationwide Danish Gynaecological Cancer Database (19).

The patients were subsequently divided into a first group consisting of patients undergoing surgery before the introduction of robotic minimally invasive surgery (RMIS) in their respective regions and a second group after the introduction of RMIS.

Five-year overall survival was compared by multivariate Cox proportional hazards models stratified by histopathological risk between Group $1(\mathrm{~N}=3,091)$ and Group $2(\mathrm{~N}=2,563)$ and between surgical modalities within Group 2 depending on whether the patients had performed total abdominal hysterectomy $(\mathrm{TAH})$, laparoscopic minimally invasive surgery (LMIS) and RMIS.

While the first part of the study showed that the patients of the second group had a higher overall survival than the patients of the first group, after the adoption of RMIS, a higher mortality was observed for the patients subjected to TAH compared to minimally invasive surgery and no survival differences in the groups subjected to LMIS and RMIS.

The authors have concluded that the national introduction of robotic surgery for early-stage endometrial cancer was associated with improved survival irrespective of age, BMI, ASA score, comorbidity, smoking, socioeconomic status and histopathological risk even if they were unable to control for adjuvant oncological treatment and did not have information on disease recurrences, preventing evaluation of the cancer-specific survival.

The results obtained suggest that robotic surgery is not only a safe approach in terms of surgical outcomes and postoperative complications as evidenced by previous studies, but also regarding mortality and survival.

This study once again encourages the use of robotic surgery, hence, an implementation of this kind of surgery is desirable for endometrial cancer. Furthermore, since the majority of patients with endometrial cancer often present comorbidities, this study has shown that robotic surgery is safe not only for early-stage endometrial cancer patients in general, but also for frail patients due to age, low socioeconomic status and concomitant diseases.

\section{Acknowledgments}

Funding: None.

\section{Footnote}

Provenance and Peer Review: This article is commissioned and reviewed by the Section Editor Hengwei Liu, MD, PhD (Department of Obstetrics and Gynecology, Union Hospital, Tongji Medical College, Huazhong University of Science and Technology, Wuhan, China).

Conflicts of Interest: All authors have completed the ICMJE uniform disclosure form (available at http://dx.doi. org/10.21037/tcr.2019.08.27). The authors have no conflicts of interest to declare.

Ethical Statement: The authors are accountable for all aspects of the work in ensuring that questions related to the accuracy or integrity of any part of the work are appropriately investigated and resolved.

Open Access Statement: This is an Open Access article distributed in accordance with the Creative Commons Attribution-NonCommercial-NoDerivs 4.0 International License (CC BY-NC-ND 4.0), which permits the noncommercial replication and distribution of the article with the strict proviso that no changes or edits are made and the original work is properly cited (including links to both the formal publication through the relevant DOI and the license). See: https://creativecommons.org/licenses/by-nc-nd/4.0/.

\section{References}

1. O'Malley DM, Smith B, Fowler JM. The role of robotic surgery in endometrial cancer. J Surg Oncol 2015;112:761-8.

2. Walker JL, Piedmonte MR, Spirtos NM, et al. Recurrence and survival after random assignment to laparoscopy versus laparotomy for comprehensive surgical staging of uterine cancer: Gynecologic Oncology Group LAP2 Study. J Clin Oncol 2012;30:695-700.

3. Minig L, Achilarre MT, Garbi A, et al. Minimally Invasive Surgery to Treat Gynecological Cancer: Conventional Laparoscopy and/or Robot-Assisted Surgery. Int J Gynecol Cancer 2017;27:562-74. 
4. Cianci S, Rosati A, Rumolo V, et al. Robotic Single-Port Platform in General, Urologic, and Gynecologic Surgeries: A Systematic Review of the Literature and Meta-analysis. World J Surg 2019;43:2401-19.

5. Lawrie TA, Liu H, Lu D, et al. Robot-assisted surgery in gynaecology. Cochrane Database Syst Rev 2019;4:CD011422.

6. Rabinovich A. Minimally invasive surgery for endometrial cancer. Curr Opin Obstet Gynecol 2015;27:302-7.

7. Park DA, Lee DH, Kim SW, et al. Comparative safety and effectiveness of robot-assisted laparoscopic hysterectomy versus conventional laparoscopy and laparotomy for endometrial cancer: a systematic review and meta-analysis. Eur J Surg Oncol 2016;42:1303-14.

8. Ind T, Laios A, Hacking $M$, et al. A comparison of operative outcomes between standard and robotic laparoscopic surgery for endometrial cancer: a systematic review and meta-analysis. Int J Med Robot 2017;13:e1851.

9. Salehi S, Avall-Lundqvist E, Legerstam B, et al. Robotassisted laparoscopy versus laparotomy for infrarenal paraaortic lymphadenectomy in women with high-risk endometrial cancer: a randomised controlled trial. Eur J Cancer 2017;79:81-9.

10. Mäenpää MM, Nieminen K, Tomás EI, et al. Roboticassisted vs traditional laparoscopic surgery for endometrial cancer: a randomized controlled trial. Am J Obstet Gynecol 2016;215:588.e1-7.

11. Gala RB, Margulies R, Steinberg A, et al. Society of gynecologic surgeons systematic review group. Systematic review of robotic surgery in gynecology: robotic techniques compared with laparoscopy and laparotomy. J Minim Invasive Gynecol 2014;21:353-61.

12. Chan JK, Gardner AB, Taylor K, et al. Robotic versus

Cite this article as: Corrado G, Bruni S, Vizza E. Robotic surgery in early-stage endometrial cancer. Transl Cancer Res 2019;8(Suppl 6):S573-S576. doi: 10.21037/tcr.2019.08.27 laparoscopic versus open surgery in morbidly obese endometrial cancer patients - a comparative analysis of total charges and complication rates. Gynecol Oncol 2015;139:300-5.

13. Corrado G, Vizza E, Cela V, et al. Laparoscopic versus robotic hysterectomy in obese and extremely obese patients with endometrial cancer: A multi-institutional analysis. Eur J Surg Oncol 2018;44:1935-41.

14. Cardenas-Goicoechea J, Shepherd A, Momeni M, et al. Survival analysis of robotic versus traditional laparoscopic surgical staging for endometrial cancer. Am J Obstet Gynecol 2014;210:160.e1-11.

15. Kilgore JE, Jackson AL, Ko EM, et al. Recurrence-free and 5-year survival following robotic-assisted surgical staging for endometrial carcinoma. Gynecol Oncol 2013;129:49-53.

16. Scalici J, Laughlin BB, Finan MA, et al. The trend towards minimally invasive surgery (MIS) for endometrial cancer: an ACS-NSQIP evaluation of surgical outcomes. Gynecol Oncol 2015;136:512-5.

17. Zanagnolo V, Achilarre MT, Maruccio M, et al. Might robotic-assisted surgery become commonplace in endometrial cancer treatment? Expert Rev Anticancer Ther 2018;18:507-9.

18. Corrado G, Cutillo G, Pomati G, et al. Surgical and oncological outcome of robotic surgery compared to laparoscopic and abdominal surgery in the management of endometrial cancer. Eur J Surg Oncol 2015;41:1074-81.

19. Jørgensen SL, Mogensen O, Wu CS, et al. Survival after a nationwide introduction of robotic surgery in women with early-stage endometrial cancer: a population-based prospective cohort study. Eur J Cancer 2019;109:1-11. 